\title{
A New Design Method and Half-Step Operation for Ultrasonic Stepping Motors
}

\author{
K. T. Chau, Member, IEEE, Bin Shi, and Min-Qiang Hu
}

\begin{abstract}
In this paper, a systematic design method and a new half-step operation are proposed for ultrasonic stepping motors (USMs) which are based on the principle of spatially shifted standing vibrations. With emphasis on the combination of the order of vibration mode, the number of spatial phase shifts, the number of electrodes, and the number of simultaneously excited electrodes, the design equations and constraints are newly derived, hence, proposing the design procedure. The finite-element method and impedance measurement are also employed to investigate the vibration of the stator. By borrowing the control idea of electromagnetic stepping motors, the half-step operation is newly applied to this USM so that the step size can be further halved. An 80-step USM is prototyped. The proposed design method and half-step operation are well supported by experimentation.
\end{abstract}

Index Terms-Piezoelectric motor, stepping motor, ultrasonic motor (USM).

\section{INTRODUCTION}

$\mathbf{T}$ HE traveling-wave ultrasonic motor (TWUM) has become attractive for servo applications because of its advantages of high torque at low speeds, light weight, compact size, fast response, no electromagnetic interference (EMI), and quiet operation [1], [2]. Nevertheless, the TWUM needs a closed-loop system with precision sensing devices [3]-[6]. In order to get rid of this bulky and costly closed-loop system, the concept of ultrasonic stepping motors (USMs) was proposed [7], [8]. However, these USMs generally involve many projections on the stator and many slits in the rotor as well as the difficulty in controlling the degenerated modes.

In [9], a USM using the principle of spatially shifted standing vibrations has been proposed in which the stator does not have any projections. However, the design of the electrode pattern for this USM has been ad hoc. There are different operation cases and different combinations of design parameters for a desired number of steps. Most of them cannot even meet the design requirements. Therefore, it is highly desirable to determine which operation case is appropriate and which combination is optimum. Increasingly, due to the segmentation problem

Paper IPCSD 03-065, presented at the 2002 Industry Applications Society Annual Meeting, Pittsburgh, PA, October 13-18, and approved for publication in the IEEE TRANSACTIONS ON INDUSTRY APPLICATIONS by the Electric Machines Committee of the IEEE Industry Applications Society. Manuscript submitted for review November 1, 2002 and released for publication May 7, 2003. This work was supported by the Hong Kong Research Grants Council.

K. T. Chau and B. Shi are with the Department of Electrical and Electronic Engineering, The University of Hong Kong, Hong Kong (e-mail: ktchau@eee.hku.hk; binshi@eee.hku.hk).

M.-Q. Hu is with the Department of Electrical Engineering, Southeast University, Nanjing 210096, China (e-mail: mqhu@ seu.edu.cn).

Digital Object Identifier 10.1109/TIA.2003.814549 of piezoelectric materials which are usually made of lead zirconate titanate (PZT), the initial number of steps is physically limited.

The purpose of this paper is to newly propose a systematic design method for USMs using spatially shifted standing vibrations. Different operation cases will be analyzed so that the design equations and constraints will be derived. In order to further halve the step size, the idea of half-step operation adopted by electromagnetic stepping motors will be newly applied to USMs. For exemplification, a new 80-step USM will be designed, and then controlled for half-step operation. The resulting prototype will be tested for verification.

In Section II, the operating principle of the USM using spatially shifted standing vibrations will be briefly described. Then, the design method, including the design equations and procedure, will be presented in Section III. Section IV will be devoted to discussing the implementation of a newly designed 80 -step prototype. The stepping control, including both full-step and half-step operations, will be discussed in Section V. In Section VI, and experimental results of the prototype will be given to verify the proposed design method and half-step operation. Finally, conclusions will be drawn in Section VII.

\section{OPERATING PRINCIPLE}

This type of USM works in such a way that a traveling wave in the stator drives the rotor to move while a standing wave in the stator controls the rotor to stop at a desired position [10]. Notice that the mechanism uses the traveling-wave contact of the vibration to cause mechanical frictional contact forces to rotate the rotor, whereas the node of vibration does not create motion. Since it is well known that a small object put on a vibrating plate moves to the nodal position of the vibration and rests at that point, this phenomenon can be utilized to visualize the vibration pattern of the USM [9], [11]. This analogy is helpful in understanding the use of spatially shifted vibrations for operation. Fig. 1 shows the step movement realization of the USM based on a spatial phase. The electrodes with the same letter identifier are connected together as one phase. It can be seen that when phase $A$ is activated by an ac voltage, a standing-wave vibration (indicated by a solid curve) is excited so that the small object rests at the nodal point (for example, point $\mathrm{P}_{1}$ ). When the excitation is switched to the phase $B$, a spatial phase shift of the standing-wave vibration (indicated by a dashed curve) occurs. Thus, the small object moves from the point $P_{1}$ to the point $P_{2}$, and takes a rest until another phase shift is turned up. The spatial phase shift corresponds to the step angle of the motor. By exciting the phases in sequence, the small object rotates step by step along the annular stator surface. In a practical USM, the 


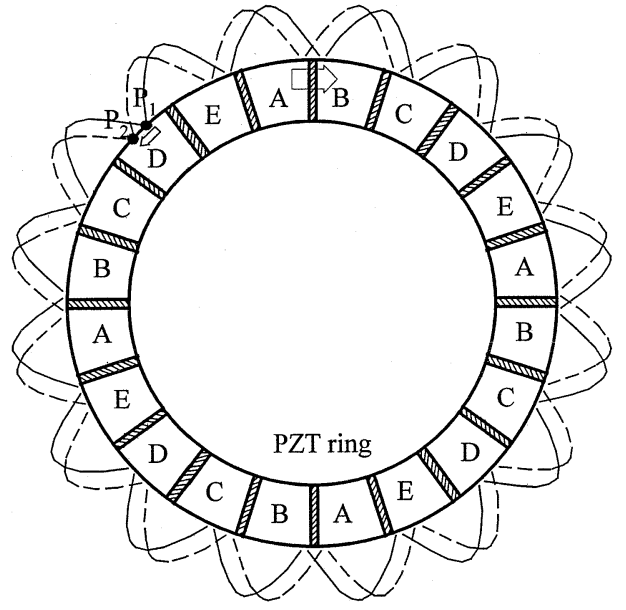

Fig. 1. Step movement realization based on spatial phase shift.

blades of the rotor act as the small objects, which are in contact with the stator surface at points. The direction of the motion can be reversed by simply changing the phase-shift sequence.

\section{DESIGN METHOD}

In the following design process, there is a basic assumption that only the desired vibration mode is excited to create motion. Practically, due to the imperfection of materials and the discrepancy of dimensions, many other vibration modes certainly exist and may affect the motion properties. To alleviate the effect of those undesirable vibration modes, two measures can be adopted. First, both the structure and dimensions of the stator should be carefully selected so as to make the resonant frequency of the desired vibration mode far away from the resonant frequencies of those undesirable vibration modes. Second, the location of electrodes should be carefully arranged to match with the desired vibration mode. In fact, the validity of this assumption is well supported by the measured vibration distribution using a laser Doppler vibrometer which will be presented in Section VI.

To design the USM, the stator and rotor structures need to be determined first. Since the corresponding dimensions can be borrowed from a traditional TWUM, the design is mainly focused on determining the electrode pattern of the stator and the number of blades on the rotor. Since the space between the blades of the rotor should be a half-wavelength of the stator vibration, the number of blades depends on the vibration mode of the stator. For a given vibration mode, the key is to design the electrode pattern of the stator, namely, the optimum combination of the order of vibration mode $n$, the number of spatial phase shifts (equivalent to the number of driving phases) $p$, the number of electrodes $q$, and the number of simultaneously excited electrodes $m$. Fig. 2 shows a general configuration of the electrode pattern in which there are $q$ electrodes (numbered from 1 to $q$ ), $p$ phases of sources (denoted from $\mathrm{S}_{1}$ to $\mathrm{S}_{p}$ ) and $p$ sets of $m$ interconnected electrodes (denoted as $\mathrm{E}_{11}, \ldots, \mathrm{E}_{1 m}$, $\mathrm{E}_{21}, \ldots, \mathrm{E}_{2 m}$ and $\left.\mathrm{E}_{p 1}, \ldots, \mathrm{E}_{p m}\right)$.

Taking into account the principle of moving object by the spatial phase shift, the ring-type stator with $B_{0 n}$ vibration mode is adopted. This $B_{0 n}$ denotes that there are no nodal circle and

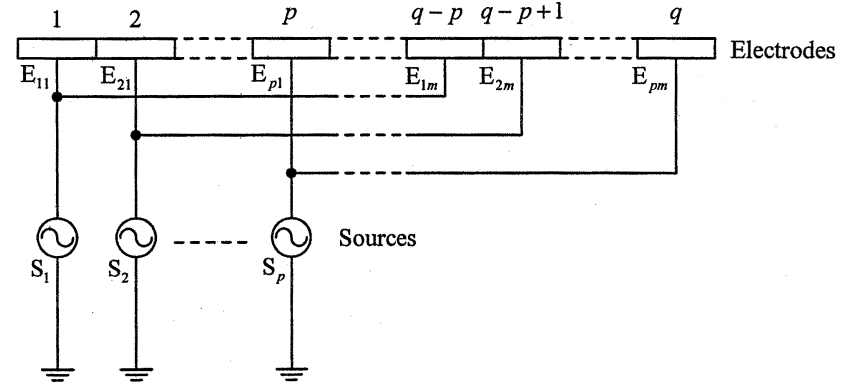

Fig. 2. General configuration of electrode pattern.

$n$ nodal diameters (equivalent to $2 n$ nodal lines) along the circumference. Thus, the vibration wavelength $\lambda$ and the electrode width $w$ can be represented as $\lambda=2 \pi / n$ and $w=2 \pi / q$, respectively, whereas the step size $\theta_{s}$ can be expressed as $\theta_{s}=2 \pi / N$ for an $N$-step USM. According to the aforementioned operating principle, the spatial phase shift should be smaller than the distance between a wave crest and a node just next to it. Therefore, the largest step size is always smaller than a quarter-wavelength of the standing-wave vibration, mathematically $\theta_{s}<\lambda / 4$. Consequently, it yields

$$
N>4 n
$$

To avoid the unnecessary mechanical damping and generate the expected vibration mode, the electrode width should be less than a half-wavelength, mathematically $w<\lambda / 2$. Since $w=2 \pi / q$, it can be rewritten as

$$
q>2 n .
$$

Since there are $p$ sets of $m$ interconnected electrodes, $q$ can be expressed as

$$
q=m p .
$$

Also, $m$ should be arranged symmetrically around the stator circumference, and needs to be an integer divisor of $n$ so as to match with the vibration mode.

Based on the relationship between the electrode width and the vibration wavelength, there are two different operation cases as shown in Fig. 3. When $w>\lambda / 4$, it is the case of small step size operation. On the contrary, when $w \leq \lambda / 4$, it is the case of large step size operation.

\section{A. Small Step Size}

Fig. 3(a) shows the operating principle at the condition of $w>\lambda / 4$. Since $w=2 \pi / q$ and $\lambda=2 \pi / n$, this condition can also be expressed as $q<4 n$. When the driving source is switched from phase $A$ to phase $B$, the spatial phase of the standing-wave vibration is shifted from the position indicated by the solid curve to the position by the dashed curve. The object on the surface moves from the node $P_{1}$ to the node $P_{2}$ at the same time. The direction of the movement is opposite to the shift of the driving phase. As shown in Fig. 3(a), the step size from node $\mathrm{P}_{1}$ to node $\mathrm{P}_{2}$ can be easily determined as given by

$$
\theta_{s}=\frac{\lambda}{2}-w
$$




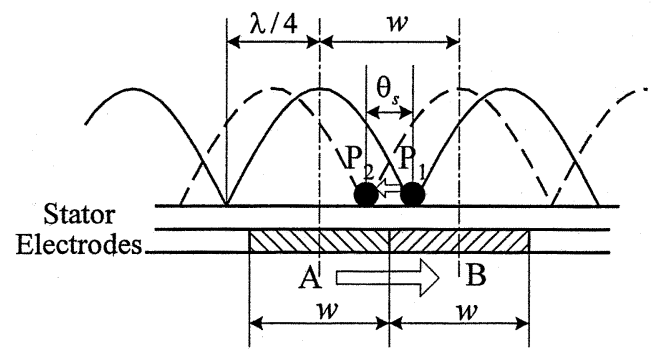

(a)

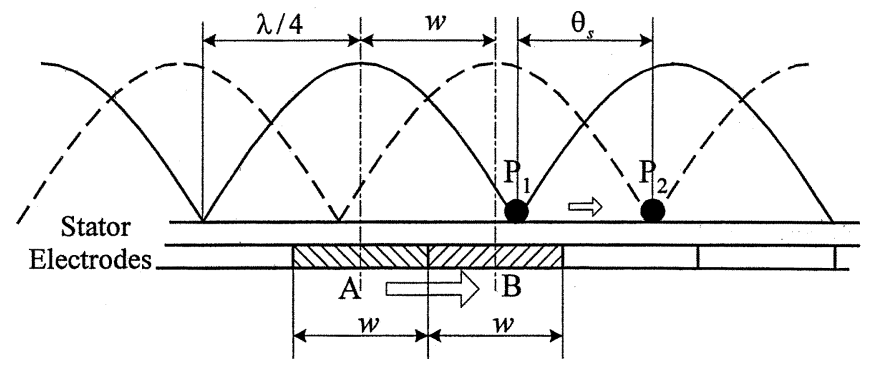

(b)

Fig. 3. Two operation cases. (a) Small step size. (b) Large step size.

Substituting $\theta_{s}=2 \pi / N, w=2 \pi / q$, and $\lambda=2 \pi / n$ into (4), $q$ can be expressed in terms of $n$ and $N$ as given by

$$
q=\frac{2 n N}{N-2 n} \text {. }
$$

Since the corresponding derivative of $q$ with respect to $n$ is always positive, there is only one value of $q$ satisfying the $n$th order vibration.

As shown in Fig. 3(a), after $p$ times switching, phase $A$ is excited again and the standing-wave vibration is again indicated by the solid curve. Therefore, the object moves from the previous node to a neighboring node of the same standing-wave vibration, namely, the object moves a distance of an integer multiple of $\lambda / 2$. On the other hand, after $p$ times switching, the object moves a distance of $p \theta_{s}$. Thus, by equating $p \theta_{s}$ to the integer multiple of $\lambda / 2$, the following relationships can be obtained:

$$
\begin{cases}N=2 n p, & \text { for } \frac{\lambda}{2} \\ N=n p, & \text { for } \lambda \\ N=\frac{2 n p}{3}, & \text { for } \frac{3 \lambda}{2} \\ N=\frac{n p}{2}, & \text { for } 2 \lambda .\end{cases}
$$

After rearrangement, (6) can be rewritten as

$$
\begin{cases}p=\frac{N}{2 n}, & \text { for } \frac{\lambda}{2} \\ p=\frac{N}{n}, & \text { for } \lambda \\ p=\frac{3 N}{2 n}, & \text { for } \frac{3 \lambda}{2} \\ p=\frac{2 N}{n}, & \text { for } 2 \lambda .\end{cases}
$$

In practice, the number of phases should be selected as small as possible to simplify the driving and control circuits. Therefore, (7) can be reduced as

$$
p=\frac{N}{2 n} .
$$

From (3), the corresponding condition to select $m$ is given by

$$
m=\frac{q}{p} \text {. }
$$

TABLE I

Design of 60-STEP USM Using Proposed Design Method

\begin{tabular}{ccccccc}
\hline$n$ & $q$ & $p$ & $m$ & $q<4 n$ & $q=\frac{2 n N}{N-2 n}$ & $\begin{array}{c}\text { Actual } \\
\text { steps }\end{array}$ \\
\hline 1 & 30 & 30 & 1 & NO & NO & 30 \\
2 & 15 & 15 & 1 & NO & NO & 15 \\
2 & 30 & 15 & 2 & NO & NO & 30 \\
3 & 10 & 10 & 1 & YES & NO & 15 \\
3 & 30 & 10 & 3 & NO & NO & 30 \\
5 & 30 & 6 & 5 & NO & NO & 30 \\
6 & 15 & 5 & 3 & YES & YES & 60 \\
6 & 30 & 5 & 6 & NO & NO & 30 \\
10 & 30 & 3 & 10 & YES & YES & 60 \\
\hline
\end{tabular}

Based on (5), (8), and (9), there is only one set of $q, p$, and $m$ for a particular $n$th vibration mode satisfying the desired $N$. For example, due to the lack of systematic design method, Nakamura [9] proposed nine possible combinations of $n, q$, and $p$ (shown in Table I) for a 60-step USM. However, based on the proposed design equations, only two combinations ( $n=6$, $q=15$, and $p=5 ; n=10, q=30$, and $p=3$ ) are appropriate. The other seven combinations cannot offer the desired number of steps. The actual numbers of steps for all nine combinations are also listed in Table I. The major reason for this incorrect design is a result of ignoring the other operation case as illustrated below.

\section{B. Large Step Size}

Fig. 3(b) shows the operating principle at the condition of $w \leq \lambda / 4$ or $q \geq 4 n$. When the driving source is switching from phase $A$ to phase $B$, the spatial phase of the standing-wave vibration is shifted and, hence, the object moves at the same time. Different from the previous operation case, the direction of the movement is the same as the shift of the driving phase. As shown in Fig. $3(\mathrm{~b})$, the step size from node $\mathrm{P}_{1}$ to node $\mathrm{P}_{2}$ can be easily determined as

$$
\theta_{s}=w
$$

Substituting $\theta_{s}=2 \pi / N$ and $w=2 \pi / q$ into (10), $q$ can be expressed as

$$
q=N
$$

As shown in Fig. 3(b), after $p$ times switching, the object moves a distance of an integer multiple of $\lambda / 2$. Also, the object moves a distance of $p \theta_{s}=2 \pi / m$. Since $m$ is an integer divisor of $n$ and $\lambda=2 \pi / n, p \theta_{s}$ becomes an integer multiple of $\lambda$. Thus, by combining both movement distances, the following relationships can be obtained:

$$
\left\{\begin{array}{ll}
N=n p, & \text { for } \lambda \\
N=\frac{n p}{2}, & \text { for } 2 \lambda
\end{array} .\right.
$$

Hence, $p$ can be obtained as

$$
\left\{\begin{array}{ll}
p=\frac{N}{n}, & \text { for } \lambda \\
p=\frac{2 N}{n}, & \text { for } 2 \lambda
\end{array} .\right.
$$

Similarly to the previous case, (13) is practically reduced as

$$
p=\frac{N}{n} \text {. }
$$


TABLE II

COMPARISON OF TWO OPERATION CASES

\begin{tabular}{cc|cc|cc}
\hline & & \multicolumn{2}{|c|}{ Small step case } & \multicolumn{2}{c}{ Large step case } \\
$N$ & $n$ & $q$ & $p$ & $q$ & $p$ \\
\hline 20 & 2 & 5 & 5 & 20 & 10 \\
30 & 5 & 15 & 3 & 30 & 6 \\
48 & 8 & 24 & 3 & 48 & 6 \\
60 & 6 & 15 & 5 & 60 & 10 \\
80 & 8 & 20 & 5 & 80 & 10 \\
\hline
\end{tabular}

TABLE III

DESIGN OF USM FROM 20 TO 100 STEPS

\begin{tabular}{ccccc}
\hline$N$ & $n$ & $q$ & $p$ & $m$ \\
\hline 20 & 2 & 5 & 5 & 1 \\
24 & 4 & 12 & 3 & 4 \\
30 & 5 & 15 & 3 & 5 \\
36 & 6 & 18 & 3 & 6 \\
40 & 4 & 10 & 5 & 2 \\
42 & 3 & 7 & 7 & 1 \\
42 & 7 & 21 & 3 & 7 \\
48 & 8 & 24 & 3 & 8 \\
54 & 9 & 27 & 3 & 9 \\
60 & 6 & 15 & 5 & 3 \\
60 & 10 & 30 & 3 & 10 \\
66 & 11 & 33 & 3 & 11 \\
72 & 4 & 9 & 9 & 1 \\
72 & 12 & 36 & 3 & 12 \\
78 & 13 & 39 & 3 & 13 \\
80 & 8 & 20 & 5 & 4 \\
84 & 6 & 14 & 7 & 2 \\
84 & 14 & 42 & 3 & 14 \\
90 & 15 & 45 & 3 & 15 \\
96 & 16 & 48 & 3 & 16 \\
100 & 10 & 25 & 5 & 5 \\
\hline
\end{tabular}

From (3), (11), and (14), the corresponding $m$ is given by

$$
m=n \text {. }
$$

It should be noted that almost all the incorrect designs tabulated in Table I correspond to this operation case, which are governed by the condition of $q \geq 4 n$ and offer the unique feature of $q=N$.

\section{Comparison of Two Operation Cases}

Theoretically, the design of the USM can be based on the above two operation cases. However, the design based on the large step size operation case has lower practicability than the small step size operation case. The reason is due to the feature of $q=N$ which results in a large number of electrodes, hence, causing a segmentation problem of small piezoelectric sections and an interconnection problem of those electrodes. Comparing the two operation cases with the same $N$ and $n$, it can be found from Table II that the design based on the large step size case needs twice the number of phases. Thus, the corresponding driving circuitry becomes more complicated and costly. Therefore, the design of the USM for the large step size operation case will be used only for some special niches such as the requirement of odd step numbers which cannot be implemented by the small step size operation case.

\section{Design Procedure}

There are six major steps constituting the design procedure of the USM using spatially shifted standing vibrations.

Step 1) The desired value $N$ is given. The initial value of $n$ is set to 1 because a lower order of vibration mode usually causes a larger amplitude of vibration and, hence, a higher stepping torque.

Step 2) If (1) is not satisfied, there is no combination of $q$, $p$, and $m$ for small step size operation. Then, the process jumps to Step 4) to consider for large step size operation. Otherwise, the process continues to Step 3).

Step 3) By using (5), (8), and (9) for small step size operation, $q, p$, and $m$ are calculated. If these values are all integers and $m$ is a factor of $n$, the desired combination is obtained and then the process is completed. Otherwise, after increasing $n$ by 1 , the process jumps back to Step 2).

Step 4) After resetting $n$ to 1, the process continues to Step 5).

Step 5) If (1) is not satisfied, there is no combination of $q$, $p$, and $m$ for large step size operation. The whole process is terminated. Otherwise, the process continues to Step 6).

Step 6) By using (11), (14), and (15) for large step size operation, $q, p$, and $m$ are calculated. If these values are all integers and $p$ is not 1 , the desired combination is obtained and the process is completed. Otherwise, after increasing $n$ by 1 , the process jumps back to Step 5).

By using the above design procedure, the optimal combinations of $n, q, p$, and $m$ for different $N$-step USMs can readily be determined. Table III shows the corresponding optimal values with $N$ ranging from 20 to 100 . It should be noted that all these combinations are determined based on the small step size operation case, since the odd number of steps is seldom considered.

\section{PROTOTYPE}

Based on the proposed design method, an 80-step USM is used for exemplification. From Table III, the optimal combination is $n=8, q=20, p=5$, and $m=4$. The corresponding structure is shown in Fig. 4, and the geometric parameters are tabulated in Table IV. Since the elastic modulus of phosphor bronze is insensitive to the varying temperature, it is selected as the material of the elastic ring of the stator [12]. The rotor is made of aluminum because of the features of lightweight and easy machining. It consists of 16 blades machined at the contact surface with the stator. The elastic caoutchouc between the flange of the shaft and the rotor acts as a fine-tuned spring. The prototype of this 80-step USM is shown in Fig. 5.

In order to estimate the appropriate driving frequency to excite the desired vibration, a vibration mode analysis is conducted. A finite-element method (FEM) based on the commercially available software ANSYS is utilized. The analysis result is depicted in Fig. 6 . It can be seen that $B_{08}$ vibration mode is implemented, and the calculated resonant frequency is $44.9 \mathrm{kHz}$. To further improve the driving effectiveness of the 


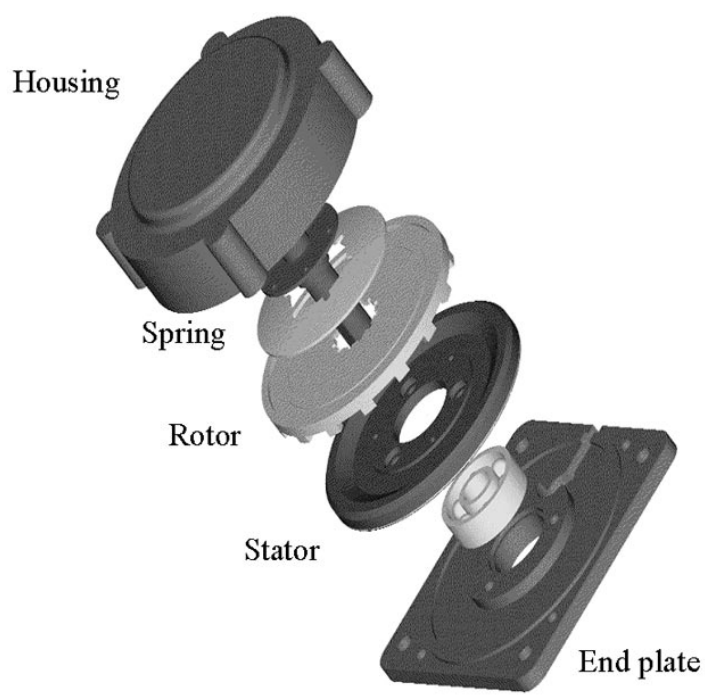

Fig. 4. Structure of USM.

TABLE IV

PHYSICAL PARAMETERS OF PROTOTYPE

\begin{tabular}{cc}
\hline Inner radius of stator & $22.5 \mathrm{~mm}$ \\
Outer radius of stator & $30.0 \mathrm{~mm}$ \\
Height of stator & $3.0 \mathrm{~mm}$ \\
Height of PZT sections & $0.5 \mathrm{~mm}$ \\
\hline
\end{tabular}

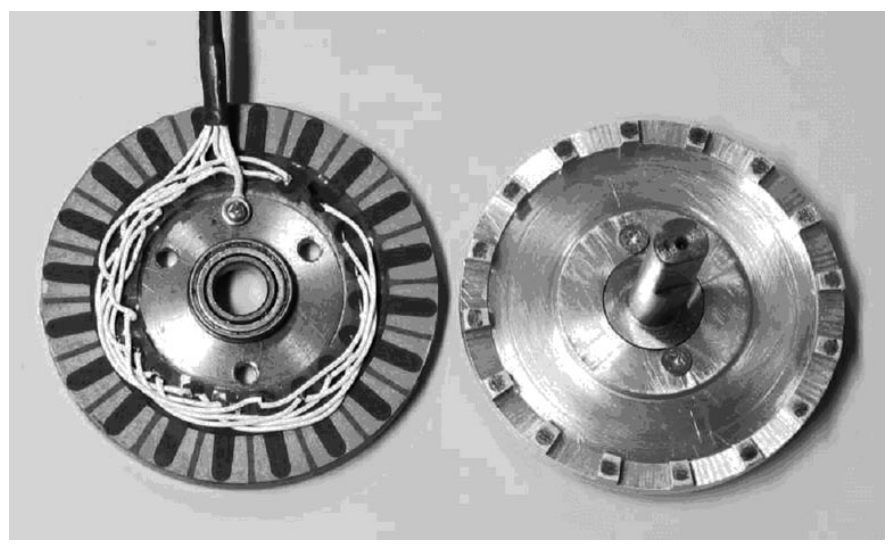

Fig. 5. Prototype of 80-step USM (left: stator; right: rotor).

USM, the driving frequency needs to be accurately determined. According to the equivalent circuit of ultrasonic motors [13], the resonant frequency can be obtained by the impedance measurement of the stator. Fig. 7 shows the recorded impedance of the stator. It can be found that the minimum value of the impedance occurs at the resonant frequency of $40.36 \mathrm{kHz}$.

Compared with the driving circuitry of a TWUM, the corresponding circuitry of the USM is much simpler. Its driving frequency can simply be generated by a voltage-controlled oscillator (VCO). Then, the generated signal is modulated by a ring counter to feed different phase channels sequentially. Thus, the rotational direction of the USM can be easily controlled by the counter direction.

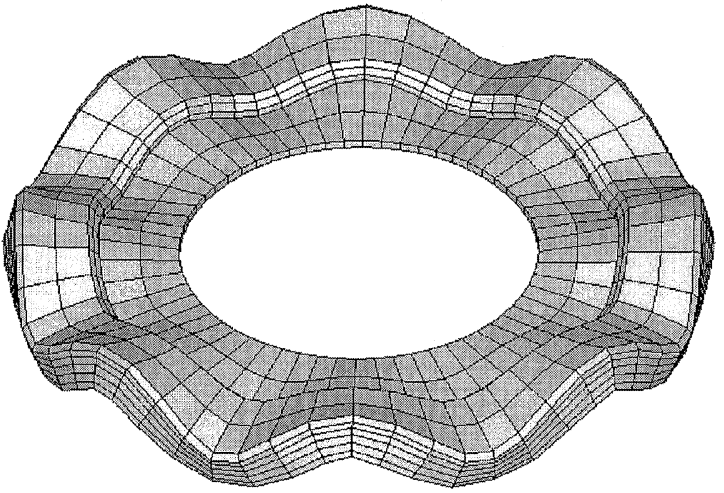

Fig. 6. Vibration mode analysis using FEM.

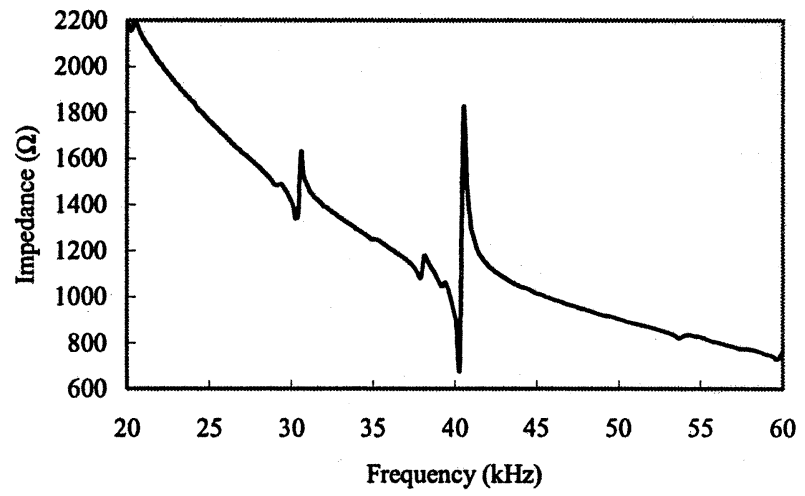

Fig. 7. Measured stator impedance.

\section{STEPPING CONTROL}

This USM has an important feature in that the principle of stepping control is very similar to that of a traditional electromagnetic stepping motor. namely, one driving pulse, one moving step. The key difference is that the USM is excited by a voltage pulse to create piezoelectric force, whereas the traditional stepping motor is excited by a current pulse to create electromagnetic force. Therefore, it is anticipated that various stepping control schemes adopted by the traditional electromagnetic stepping motors can be extended to this USM.

It is well known that the traditional electromagnetic stepping motor can offer the half-step operation by combining the single-phase and two-phase excitations. By further extending this idea, the half-step operation is newly applied to this USM. Table V shows the excitation sequences for the normal full-step operation (the single-phase excitation is activated only) and the half-step operation (the single-phase and two-phase excitations are alternately activated).

\section{EXPERIMENTAL CHARACTERISTICS}

A measurement system is constructed to investigate the characteristics of the USM. Although this USM does not need any position feedback, a position encoder having a resolution of 2500 pulses per revolution is coupled to the USM so that the angular position of the rotor can be directly measured. The driving frequency is set to the measured resonant frequency of the stator, namely, $40.36 \mathrm{kHz}$. The driving voltage of electrodes is set to 
TABLE $\mathrm{V}$

EXCITATION SEQUENCES

Full-step operation

\begin{tabular}{|l|l|l|l|l|l|l|l|l|l|l|}
\hline Clock state & $\mathrm{R}$ & 1 & 2 & 3 & 4 & 5 & 6 & 7 & 8 & 9 \\
\hline Phase A & & & & & & & & & & \\
\hline Phase B & & & & & & & & & & \\
\hline Phase C & & & & & & & & & & \\
\hline Phase D & & & & & & & & & & \\
\hline Phase E & & & & & & & & & & \\
\hline
\end{tabular}

Half-step operation

\begin{tabular}{|l|l|l|l|l|l|l|l|l|l|l|}
\hline Clock state & R & 1 & 2 & 3 & 4 & 5 & 6 & 7 & 8 & 9 \\
\hline Phase A & & & & & & & & & & \\
\hline Phase B & & & & & & & & & & \\
\hline Phase C & & & & & & & & & & \\
\hline Phase D & & & & & & & & & & \\
\hline Phase E & & & & & & & & & & \\
\hline
\end{tabular}

$200 \mathrm{~V}$ (peak-to-peak). The sampling frequency of the position information is set to $400 \mathrm{~Hz}$.

\section{A. Static Characteristics}

To verify the spatial phase shift of the 80 -step USM, the vibration mode of the stator is measured by using a laser Doppler vibrometer. Fig. 8(a) shows the spatial distribution of the vibration when only phase $A$ is excited. The black line between two sections along the circumference denotes the nodal line at which there is no vibration. The reference position is one of these nodal lines as indicated by a dashed line marked with "Ref". When the excitation is switched from phase $A$ to phase $B$, a spatial phase shift occurs. Fig. 8(b) shows the corresponding distribution of the vibration when only phase $B$ is excited. The relevant nodal line is thus indicated by a solid line. Comparing the two patterns, the spatial phase shift is $4.5^{\circ}$ when the excitation is switched from phase $A$ to phase $B$. Therefore, the rotor also moves $4.5^{\circ}$ per step, in total, 80 steps per revolution.

\section{B. Dynamic Characteristics}

Firstly, the dynamic characteristics of the USM at no load for the normal full-step operation are investigated. When a step command of $4.5^{\circ}$ is applied, the transient position and speed responses of the rotor are recorded as shown in Fig. 9. It can be seen that the rotor rapidly increases the speed to its maximum within $25 \mathrm{~ms}$ and then slows down gradually upon approaching the desired step size. Hence, the rotor can achieve the step motion within $200 \mathrm{~ms}$. Moreover, the stepping motion characteristics of the USM under full-step operation are recorded. The corresponding position commands are to rotate ten steps in the clockwise direction, then to reverse the rotation for another ten steps, and finally to stop at its original position. As shown in Fig. 10, the rotor can accurately follow these position commands with a step size of $4.5^{\circ}$. During the whole operation, the maximum position deviation is less than $1^{\circ}$, and there is no cumulative error.

Secondly, the dynamic characteristics of the USM at no load for the half-step operation are investigated. When a step command of $2.25^{\circ}$ is applied, the transient position and speed responses of the rotor are recorded as shown in Fig. 11. As

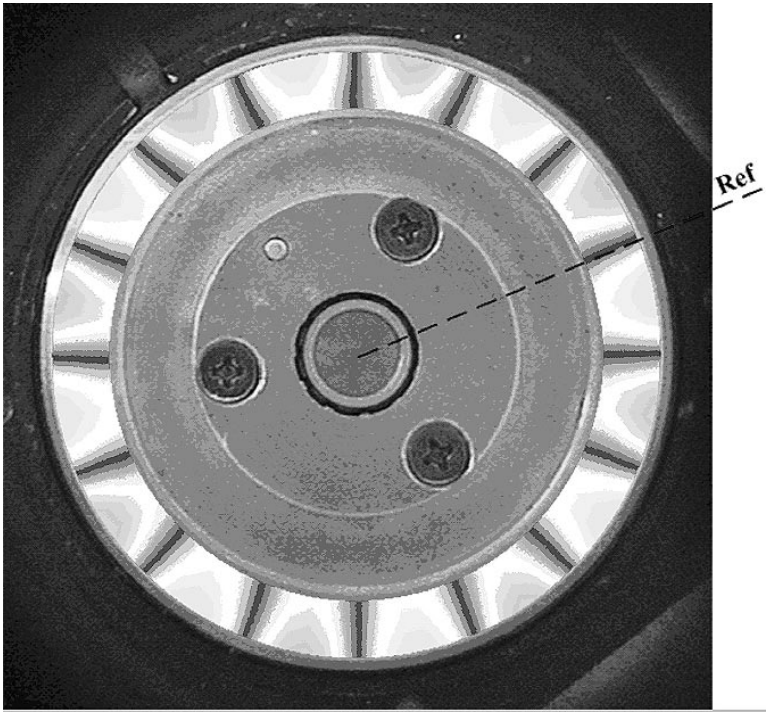

(a)

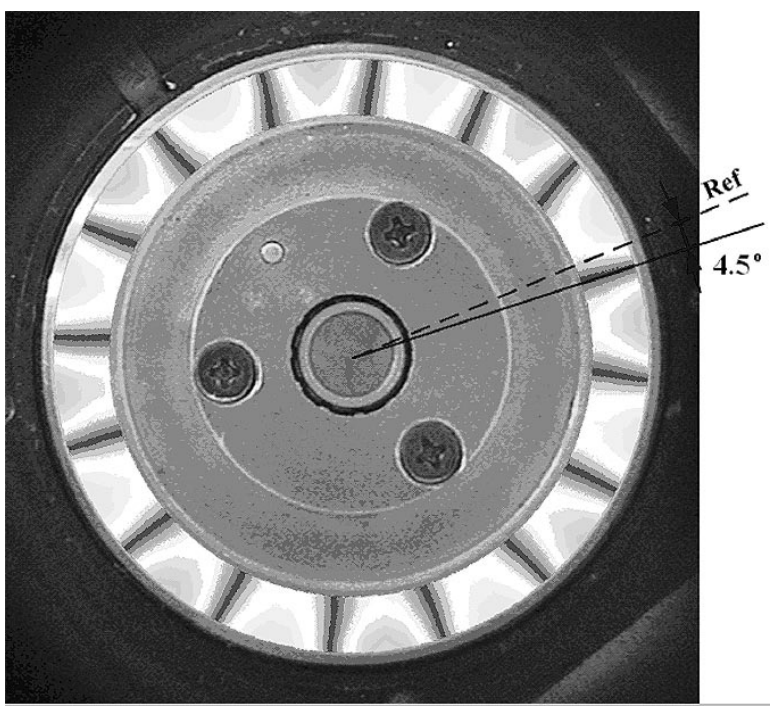

(b)

Fig. 8. Measured vibration distribution. (a) Phase $A$ excited. (b) Phase $B$ excited.

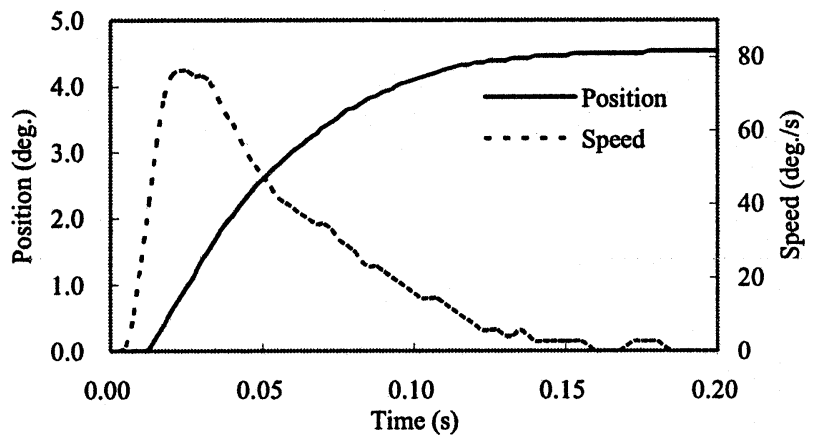

Fig. 9. Transient responses under full-step operation.

expected, the rotor rapidly increases the speed to its maximum within $18 \mathrm{~ms}$ and then slows down gradually upon approaching the desired step size. It is interesting to note that the corresponding maximum speed and the rate of speed rise are much larger than those under full-step operation. This is because 


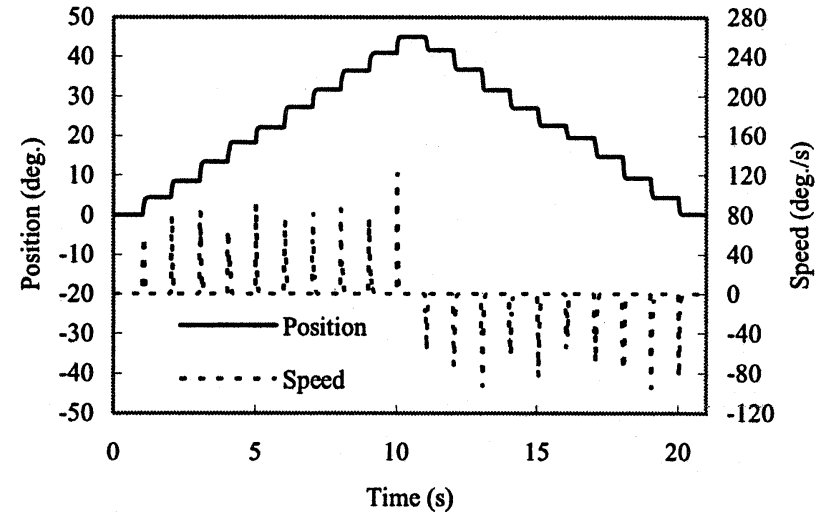

Fig. 10. Motion characteristics under full-step operation.

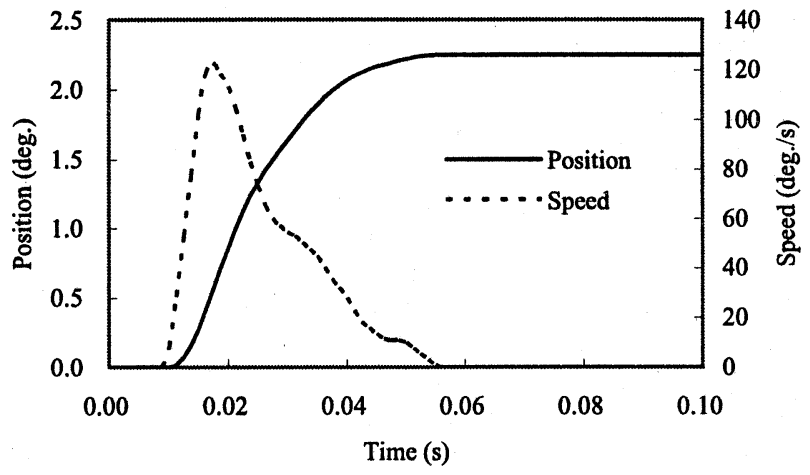

Fig. 11. Transient responses under half-step operation.

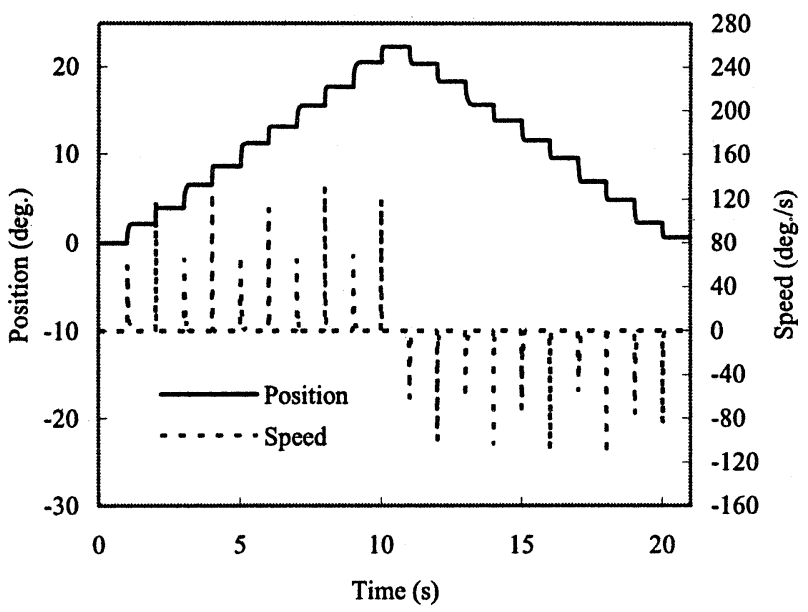

Fig. 12. Motion characteristics under half-step operation.

the switching sequence for half-step operation is between the single-phase and two-phase excitations, rather than switching between two single-phase excitations for full-step operation. Hence, the rotor can achieve a step within $60 \mathrm{~ms}$, which is much faster than that under normal full-step operation. Also, the stepping motion characteristics of the USM under half-step operation are recorded. Again, the corresponding position commands are to rotate ten steps in the clockwise direction, then to reverse the rotation for another ten steps, and finally to stop at its original position. As shown in Fig. 12, the rotor can successfully follow these position commands with a step size of $2.25^{\circ}$.

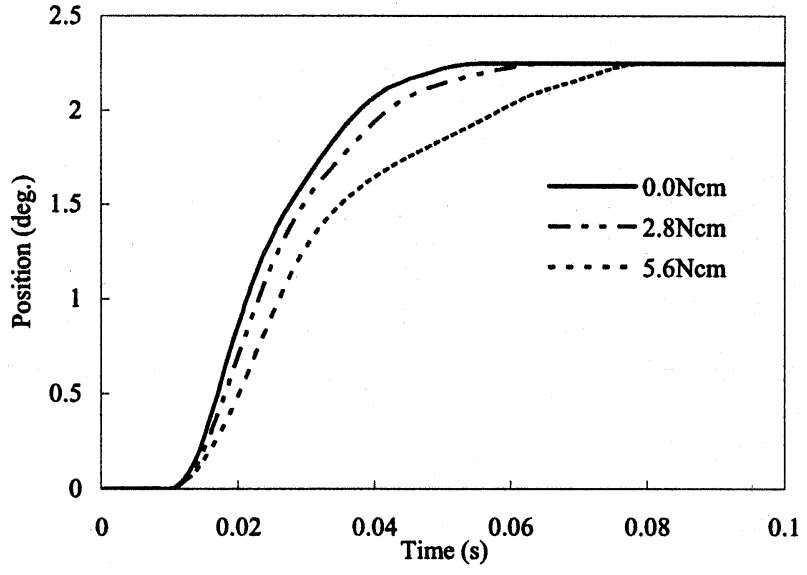

Fig. 13. Comparison of transient position responses at different loads under half-step operation.

Finally, in order to further assess the transient performance of the USM for half-step operation, three position step responses at various load torques (namely, $0,2.8$, and $5.6 \mathrm{~N} \cdot \mathrm{cm}$ ) are recorded as shown in Fig. 13. As expected, the USM can successfully offer a step size of $2.25^{\circ}$ while the transition time increases with the load torque. Based on this result, it is anticipated that the transient performance is similarly influenced by the axial preload force used in assembling the USM.

\section{CONCLUSION}

In this paper, a systematic design method and a new half-step operation have been proposed and implemented for the USM which are based on the principle of spatially shifted standing vibrations. Two operation cases, namely, small step size and large step size, have been revealed. The corresponding design equations governing the combination of the order of vibration mode, the number of spatial phase shifts, the number of electrodes, and the number of simultaneously excited electrodes have been derived, hence, proposing the design procedure. Both the FEM and impedance measurement have also been employed to determine the driving frequency of an 80-step USM prototype. Following the control idea adopted by an electromagnetic stepping motor, the half-step operation has been newly applied to this USM. The proposed design method and half-step operation have been verified by experimentation.

\section{ACKNOWLEDGMENT}

The authors would like to thank the reviewers for their thoughtful and constructive comments.

\section{REFERENCES}

[1] S. Ueha and Y. Tomikawa, Ultrasonic Motors: Theory and Applications. New York: Oxford, 1993.

[2] K. Uchino, "Piezoelectric ultrasonic motors: Overview," Smart Mater. Structures, vol. 7, no. 3, pp. 273-285, 1998.

[3] T. Senjyu, H. Miyazato, and K. Uzeato, "Quick and precise position control of an ultrasonic motor with dual mode control," Int. J. Electron., vol. 80, no. 2, pp. 191-200, 1996.

[4] F. J. Lin, "Fuzzy adaptive model-following position control for ultrasonic motor," IEEE Trans. Power Electron., vol. 12, pp. 261-268, Mar. 1997. 
[5] K. T. Chau and S. W. Chung, "Servo position control of ultrasonic motors using fuzzy neural network," Elect. Mach. Power Syst., vol. 29, no. 3, pp. 229-246, 2001.

[6] - "Servo speed control of traveling-wave ultrasonic motors using pulse width modulation," Electr. Power Comp. Syst., vol. 29, no. 8, pp. 707-722, 2001

[7] C. Kusakabe, "Effect of pressing force applied to a rotor on disk-type ultrasonic motor driven by self-oscillation," Jpn. J. Appl. Phys., pt. 1, vol. 37, no. 5B, pp. 2966-2969, 1998.

[8] S. He, W. Chen, X. Tao, and Z. Chen, "Standing wave bi-directional linearly moving ultrasonic motor," IEEE Trans. Ultrason., Ferroelect., Freq. Contr., vol. 45, pp. 1133-1139, Sept. 1998.

[9] K. Nakamura, J. Margairaz, T. Ishii, and S. Ueha, "Ultrasonic stepping motor using spatially shifted standing vibrations," IEEE Trans. Ultrason., Ferroelect., Freq. Contr., vol. 44, pp. 823-827, July 1997.

[10] Y. Chen, T. Y. Zhou, Q. Zhang, X. Y. Chen, and S. H. Chen, "A study on the friction of a self-correction ultrasonic stepping motor," Ultrasonics, vol. 39, no. 9, pp. 667-671, 2002.

[11] J. W. S. Rayleigh, The Theory of Sound. New York: Dover, 1945.

[12] T. Sashida and T. Kenjo, An Introduction to Ultrasonic Motors. New York: Oxford, 1993.

[13] N. E. Ghouti, "Hybrid modeling of a traveling wave piezoelectric motor," Doctoral dissertation, Aalborg University, Aalborg East, Denmark, 2000.

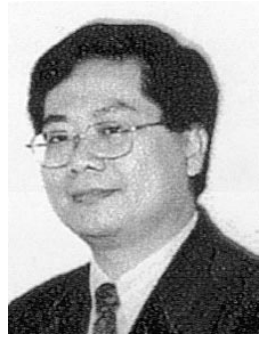

K. T. Chau (M'89) received the first-class honors B.Sc.(Eng.), M.Phil., and Ph.D. degrees in electrical and electronic engineering from The University of Hong Kong, Hong Kong, in 1988, 1991, and 1993, respectively.

He is currently an Associate Professor at The University of Hong Kong. His teaching and research interests focus on three main areas: power converters, machines and drives, and electric vehicles. In these areas, he has authored more than 100 published refereed technical papers and several industrial reports. He has also served as chair and organizing committee member for many international conferences. He is the coauthor of a monograph, Modern Electric Vehicle Technology (London, U.K.: Oxford Univ. Press, 2001).

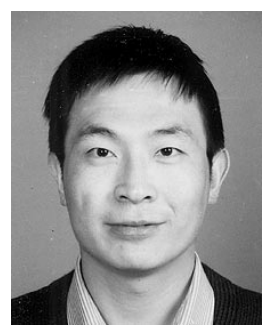

Bin Shi was born in Shanxi, China, in 1973. He received the B.Sc.(Eng.) degree and Ph.D. degree in electrical engineering from Southeast University, Nanjing, China, in 1995 and 2001, respectively.

$\mathrm{He}$ is currently a Research Associate in the Department of Electrical and Electronic Engineering, The University of Hong Kong, Hong Kong. His current research interests include motor control, ultrasonic motor modeling, and power electronics.

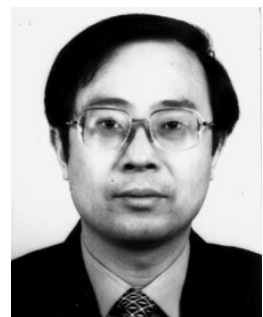

Min-Qiang Hu was born in Jiangsu, China, in 1961. He received the B.Sc.(Eng.) and M.Phil. degrees in electrical power systems and automation from Tianjin University, Tianjin, China, in 1982 and 1985, respectively, and the Ph.D. degree in electrical engineering from Huazhong University of Technology, Wuhan, China, in 1989.

In 1989, he joined the Department of Electrical Engineering, Southeast University, Nanjing, China, where he has served as Head of the Department. He is currently a Professor, Vice Dean of the Faculty of the Graduate School, and Assistant Chancellor. His main research areas include electromagnetic field theory, electric motors, and electric energy utilization. $\mathrm{He}$ is the holder of several patents, has authored over 90 publications, and has delivered many presentations.

Dr. Hu is currently a member of the Technology Division of the Science and Technology Committee in the Ministry of Education, a Council Member of the Chinese Electrical Engineering Association, and Director of the Jiangsu Electrical Technology Institute. 